\title{
Blood levels of dual-specificity phosphatase-1 independently predict risk for post-operative morbidities causing prolonged hospitalization after coronary artery bypass grafting
}

\author{
SARA HÄGG ${ }^{1,4}$, THOMAS ALSERIUS ${ }^{2}$, PERI NOORI ${ }^{1,3}$, ARNO RUUSALEPP $^{5}$, TORBJÖRN IVERT ${ }^{2}$, \\ JESPER TEGNÉR ${ }^{3,4}$, JOHAN BJÖRKEGREN ${ }^{1,4,5^{*}}$ and JOSEFIN SKOGSBERG ${ }^{1,4^{*}}$

\begin{abstract}
${ }^{1}$ The Cardiovascular Genomics Group, Department of Medical Biochemistry and Biophysics, Solna; ${ }^{2}$ Department of Cardiothoracic Surgery and Anaesthesiology and Department of Molecular Medicine and Surgery, Karolinska University Hospital Solna; ${ }^{3}$ Computational Medicine, Center for Molecular Medicine, Department of Medicine, Solna, Karolinska Institutet, Stockholm; ${ }^{4}$ Clinical Gene Networks, Fogdevreten 2b, Stockholm, Sweden;
\end{abstract} \\ ${ }^{5}$ Department of Cardiac Surgery, Tartu University and University Hospital, Tartu, Estonia
}

Received December 22, 2010; Accepted January 28, 2011

DOI: $10.3892 /$ ijmm.2011.650

\begin{abstract}
New technologies to generate high-dimensional data provide unprecedented opportunities for unbiased identification of biomarkers that can be used to optimize pre-operative planning, with the goal of avoiding costly postoperative complications and prolonged hospitalization. To identify such markers, we studied the global gene expression profiles of three organs central to the metabolic and inflammatory homeostasis isolated from coronary artery disease (CAD) patients during coronary artery bypass grafting (CABG) surgery. A total of 198 whole-genome expression profiles of liver, skeletal muscle and visceral fat from 66 CAD patients of the Stockholm Atherosclerosis Gene Expression (STAGE) cohort were analyzed. Of $\sim 50,000$ mRNAs measured in each patient, the mRNA levels of the anti-inflammatory gene, dualspecificity phosphatase-1 (DUSP1) correlated independently with post-operative stay, discriminating patients with normal ( $\leq 8$ days) from those with prolonged ( $>8$ days) hospitalization $(\mathrm{p}<0.004)$. To validate DUSP1 as a marker of risk for post-operative complications, we prospectively analyzed 181 patients undergoing CABG at Tartu University Hospital for
\end{abstract}

Correspondence to: Dr Josefin Skogsberg or Dr Johan Björkegren, Karolinska Institutet, Department of Medical Biochemistry and Biophysics, Vascular Biology Unit, Cardiovascular Genomics Group, Scheeles väg 2, A4:02, SE-17177 Stockholm, Sweden

E-mail: josefin.skogsberg@ki.se

E-mail: johan.bjorkegren@ki.se

*Contributed equally

Key words: Coronary artery disease, post-operative stay; gene expression profiling, dual-specificity phosphatase-1, biological marker
DUSP1 protein levels in pre-operative blood samples. The pre-operative plasma levels of DUSP1 clearly discriminated patients with normal from those with prolonged hospitalization $\left(\mathrm{p}=2 \times 10^{-13}\right.$; odds ratio $=5.1, \mathrm{p}<0.0001$; receiver operating characteristic area under the curve $=0.80$ ). Taken together, these results indicate that blood levels of the anti-inflammatory protein DUSP1 can be used as a biomarker for post-operative complications leading to prolonged hospitalization after $\mathrm{CABG}$ and therefore merit further testing in longitudinal studies of patients eligible for CABG.

\section{Introduction}

New technologies to generate genome-wide molecular activity measurements, in combination with systems analyses of biological data, allow for a more complete understanding of the molecular interactions underlying complex diseases such as coronary artery disease (CAD) (1). The assembled databases can also be used to identify molecular biomarkers to predict disease development and responses to therapies and interventions (2). Surgical procedures pose a risk for post-operative morbidity. Moreover, these procedures are expensive as is the post-operative care. From the perspective of the healthcare economy as well as the well-being of the individual patient, identifying markers to predict the risk for post-operative complications is desirable.

Coronary artery bypass grafting (CABG) is a safe, widely used procedure to revascularize the myocardium in patients with obstructive CAD; however, it is associated with an increased risk of post-operative morbidities. For example, CAD patients with diabetes mellitus have an increased risk of morbidity and mortality after CABG (3-7), as do hyperglycemic patients without a clinical diagnosis of diabetes $(7,8)$. Since only a fraction of CABG patients also have diabetes, a more generally useful but specific predictor of the increased risk for prolonged post-operative stay due to morbidity after CABG would be of great clinical value. Patients at increased 
risk could be rescheduled (if CAD status allows) or undergo more extensive pre-operative and post-operative care.

In this study, we sought to identify biomarkers of postoperative morbidities leading to prolonged hospital stay after CABG. We first examined a unique compendium of 198 transcriptional profiles of liver, skeletal muscle and visceral fat isolated from 66 well-characterized patients during CABG [part of the Stockholm Atherosclerosis Gene Expression (STAGE) cohort] (9). From this cohort, we identified dualspecificity phosphatase-1 (DUSPI) as an independent marker of post-operative morbidities and hospital stay. Then, in a prospective validation study of $181 \mathrm{CABG}$ patients, preoperative DUSP1 plasma levels clearly discriminated patients who suffered post-operative morbidities leading to prolonged stay from those who had uncomplicated, normal postoperative stay.

\section{Materials and methods}

Patient selection. The STAGE cohort (9) was used to examine hospitalization or rehabilitation days in CABG patients. In brief, 124 patients undergoing CABG at the Karolinska University Hospital, Solna, were recruited. The exclusion criteria were the presence of other active severe diseases (e.g., cancer, kidney disease, and chronic systemic inflammatory diseases). The STAGE study was approved by the hospital's ethics committee. To validate the findings, we collected preoperative blood samples from 250 patients undergoing CABG at the Tartu University Hospital; approval was obtained from the ethics committees at Tartu University Hospital (Ethics Review Committee on Human Research) and in Stockholm (Etisk prövningsnämnd, Stockholm). All patients gave written informed consent. Fifty-two Tartu patients were excluded because they had prolonged post-operative hospital stays for reasons not directly linked to the surgery (e.g., social or mental, $\mathrm{n}=38$ ) or left the hospital early despite complications related to surgery $(n=7)$ or because blood samples were mistakenly drawn after surgery $(\mathrm{n}=7)$, leaving a total of 198 patients. Tissue samples were collected during CABG by four surgeons at Karolinska Hospital and three at Tartu University Hospital, who followed a strict protocol that mandated biopsy collection at specific time-points during the CABG surgery. Anesthesia was standardized; systolic blood pressure was kept at $<150 \mathrm{mmHg}$. Biopsies were obtained from the skeletal muscle, visceral mediastinal fat and the anterior hepatic edge (liver) through a small incision in the diaphragm, preserved in RNAlater (Qiagen), and frozen at $-80^{\circ} \mathrm{C}$.

Patient characterization. Using a standard questionnaire, a research nurse obtained a medical history and information on lifestyle factors (e.g., smoking, alcohol consumption, and physical activity) from 114 STAGE patients (9) 3 months after surgery and from the Tartu cohort before the surgery. All patients underwent a physical examination, and venous blood samples were drawn into precooled sterile tubes (Vacutainer, Becton-Dickinson) containing sodium EDTA and placed on ice. Plasma was recovered within $30 \mathrm{~min}$ by centrifugation $\left(2,750 \mathrm{x} \mathrm{g}, 20 \mathrm{~min}, 4^{\circ} \mathrm{C}\right)$ for analysis of cholesterol, triglyceride and lipoproteins as previously described (10). Blood glucose was measured by a glucose oxidation method (Kodak
Ektachem). In the STAGE cohort, fasting blood glucose was determined before anesthesia on the morning of surgery from whole blood by the glycodehydrogenase method (HemoCue; Ängelholm, Sweden). At Karolinska and Tartu Hospitals, all CABG patients were allowed to stay for 8 days after surgery regardless of their physical conditions. Longer hospital stays were based on the individual status of the patients. Questionnaires to assess post-operative health-related quality of life were mailed to the STAGE patients on average 3.3 years (range 2.7-4.3 years) after surgery.

Gene expression profiling in STAGE. RNA was isolated from the biopsies with TRIzol (BRL-Life Technologies) and FastPrep (MP Biomedicals), purified with the RNeasy Mini kit (Qiagen) with a DNase1 treatment step (Qiagen). Sample quality was assessed with the Agilent Bioanalyzer 2100 . cRNA yield was assessed with a spectrophotometer (ND-1000, NanoDrop Technologies) before hybridization to HG-U133 Plus 2.0 arrays (Affymetrix). The arrays were processed with a Fluidics Station 450, scanned with a GeneArray Scanner 3000, and analyzed with the GeneChip Operational software 2.0.

Reverse transcriptase-polymerase chain reaction (RT-PCR). For cDNA synthesis, $150 \mathrm{ng}$ of total RNA was reverse transcribed with Superscript III (Invitrogen) according to the manufacturer's protocol. After a 4-fold dilution, cDNA (3 $\mu \mathrm{l})$ was amplified by real-time PCR with $1 \mathrm{X}$ TaqMan universal PCR master mix (Applied Biosystems) on an ABI Prism 7000 (PE Biosystems) with software according to the manufacturer's protocol. Assay-On-Demand kits containing corresponding primers and probes from Applied Biosystems were used, and expression values were normalized to acidic ribosomal phosphoprotein P0. Each sample was analyzed in duplicate.

Enzyme-linked immunosorbent assay (ELISA). Plasma concentrations of DUSP1 in blood samples from patients in Tartu were determined by ELISA. Wells were coated overnight at $4^{\circ} \mathrm{C}$ with $100 \mu \mathrm{l}$ of diluted $(1 \mu \mathrm{g} / \mathrm{l})$ capture antibody (DUSP1; M-18, Santa Cruz Biotechnology). After washing and blocking, samples were incubated with biotinylated antibody (M-18; 1:400). Streptavidin-horseradish peroxidase (R\&D Systems) and 3,3',5,5'-tetramentylbenzidine (TMB) (Invitrogen) were used for detection. In 17 of 198 blood samples, plasma proteins had degenerated, and DUSP1 levels could not be determined, leaving a total of 181 DUSP1 measurements.

Statistical analyses. Clinical and metabolic characteristics are given as continuous variables with the means and SD or as categorical variables with numbers and percentages of subjects. Gene expression values were pre-processed with Quantile Normalization and the Robust Multichip Average (11). Of 604,258 perfect-match Affymetrix probe signals, 280,523 could be mapped to 16,685 Entrez genes without using cross-hybridizing probes. P-values were calculated for continuous variables with unpaired t-tests; skewed values were log-transformed. Spearman rank correlation was used for correlation analysis; $\mathrm{p}$-values were determined by a t-test after a Fisher transformation. Mathematica 5.1 or StatView 5.0.1 were used for all calculations. 


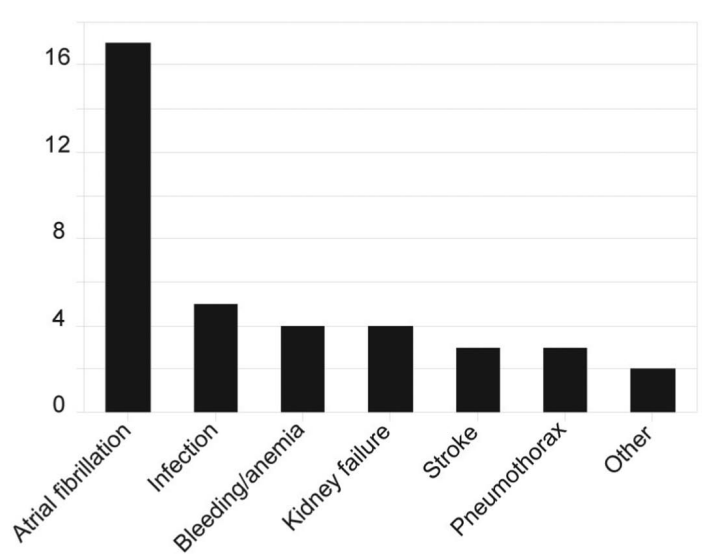

Figure 1. Complications leading to prolonged hospitalization in the STAGE cohort. Number of STAGE patients with specific post-operative CABG complications. Six patients had more than one complication.

\section{Results}

$C A B G$ morbidities causing prolonged hospitalization in STAGE patients. The characteristics of the STAGE cohort are shown in Table I. Their age, gender distribution, and drug therapy were typical for CABG cohorts. The most frequent reason for prolonged hospitalization ( $>8$ days) was atrial fibrillation, followed by post-operative infections, bleeding, kidney failure, stroke, and pneumothorax (Fig. 1). Of the 37 patients with prolonged hospitalization, all but 4 had at least one of these CABG morbidities (Fig. 1), suggesting that the reasons for prolonged hospitalization were directly linked to complications of CABG.

The duration of the post-operative stay did not correlate with any of the clinical characteristics of the patients (Table I), although trends were observed for age at surgery $\left(r^{2}=0.21 ; p=0.09\right)$ and pre-operative fasting blood glucose levels $\left(r^{2}=0.24 ; p=0.06\right)$. The borderline significance of the correlation with fasting blood glucose levels is consistent with previous studies that diabetics undergoing CABG suffer more post-operative morbidities, leading to prolonged hospitalization $(3,7,8,12,13)$. Importantly, post-operative stay did not correlate with the extent of coronary stenosis, as judged from pre-operative angiograms (not shown).

Markers of post-operative morbidities leading to prolonged hospitalization in the STAGE cohort. We first analyzed the STAGE gene expression compendium to seek changes in gene activity in the liver, skeletal muscle, and visceral fat that could predict $\mathrm{CABG}$ patients at risk of a prolonged post-operative stay, either adding days for rehabilitation ('rehabilitation') or not ('hospitalization'). To this end, we examined the correlations between the days of post-operative stay and mRNA levels obtained from each of the three tissues. Many of the correlated genes were related to immune or inflammatory processes (Table II). This is not surprising since the most common post-operative morbidities are associated with inflammatory processes $(14,15)$.

DUSPI had the highest correlation with rehabilitation days $\left(r^{2}=0.44\right)$, together with the major histocompatibility class II, DO $\beta$ (HLA-DOB) in the liver $\left(r^{2}=-0.45\right)$. However, in contrast to HLA-DOB the correlation with DUSPI was validated
Table I. Basic characteristics of the STAGE patients cohort.

\begin{tabular}{|c|c|c|}
\hline Continuous variables & Mean & SD \\
\hline Age (years) & 66 & 8 \\
\hline \multicolumn{3}{|l|}{ Pre-operatively } \\
\hline FBG $(\mathrm{mmol} / \mathrm{l})$ & 5.6 & 1.4 \\
\hline HbA1c (\%) & 5.3 & 1.1 \\
\hline \multicolumn{3}{|l|}{3 months post-operatively } \\
\hline $\operatorname{BMI}\left(\mathrm{kg} / \mathrm{m}^{2}\right)$ & 26.4 & 3.9 \\
\hline WHR & 0.93 & 0.06 \\
\hline \multicolumn{3}{|l|}{ Blood pressure $(\mathrm{mmHg})$} \\
\hline Systolic & 140 & 19 \\
\hline Diastolic & 80 & 10 \\
\hline HbA1c (\%) & 5 & 0.7 \\
\hline Creatinine $(\mathrm{mmol} / \mathrm{l})$ & 87.6 & 20.1 \\
\hline $\mathrm{CRP}(\mathrm{mg} / \mathrm{l})$ & 5.9 & 9 \\
\hline \multicolumn{3}{|l|}{ Cholesterol (mmol/l) } \\
\hline Total & 3.97 & 1.08 \\
\hline LDL & 2.01 & 0.84 \\
\hline HDL & 1.51 & 0.33 \\
\hline Total triglycerides $(\mathrm{mmol} / \mathrm{l})$ & 1.36 & 0.7 \\
\hline Alcohol consumption (g/week) & 117 & 89 \\
\hline \multicolumn{3}{|l|}{ Angiographic score } \\
\hline Mean $\%$ segment area & 7 & 1.7 \\
\hline Stenosis score & 5.06 & 2.41 \\
\hline Categorical variables & No. & $(\%)$ \\
\hline \multicolumn{3}{|l|}{ Sex } \\
\hline Male & 59 & 89 \\
\hline Female & 7 & 11 \\
\hline \multicolumn{3}{|l|}{ Smokers } \\
\hline Current & 4 & 6 \\
\hline Former & 42 & 64 \\
\hline Non-smoker & 20 & 30 \\
\hline Pre-operative heart attack & 13 & 20 \\
\hline \multicolumn{3}{|l|}{ Present disease } \\
\hline Hypertension & 43 & 65 \\
\hline Hyperlipidemia & 49 & 74 \\
\hline \multicolumn{3}{|l|}{ Post-operative dyspnea } \\
\hline Never & 25 & 38 \\
\hline Heavy effort & 14 & 21 \\
\hline Moderate effort & 20 & 30 \\
\hline Easy effort & 6 & 9 \\
\hline \multicolumn{3}{|l|}{ Treatment } \\
\hline Statins & 61 & 92 \\
\hline$\beta$-blockers & 62 & 94 \\
\hline Insulin (oral/s.c.) & 9 & 14 \\
\hline
\end{tabular}

FBG, fasting blood glucose; HbA1c, glycated haemoglobin; BMI, body mass index; WHR, waist-hip ratio; CRP, C-reactive protein; LDL, low density lipoprotein; HDL, high density lipoprotein. STAGE patients, $\mathrm{n}=66$. 
Table II. Correlations between inflammatory genes and rehabilitation.

\begin{tabular}{lcc}
\hline Gene & Correlation & p-Value \\
\hline Skeletal muscle & & 0.0002 \\
Dual specificity phosphatase 1 & 0.44 & 0.0006 \\
Zinc finger protein 36 & 0.41 & 0.002 \\
Splicing factor arginine/serine-rich 10 & 0.38 & 0.003 \\
Mitogen-activated protein kinase kinase 1 interacting protein 1 & 0.36 & 0.003 \\
Matrix metallopeptidase-like 1 & -0.36 & 0.003 \\
Natural cytotoxicity triggering receptor 1 & -0.36 & 0.004 \\
Interferon $\gamma$ receptor 1 & 0.35 & 0.004 \\
Forkhead box H1 & -0.35 & 0.0001 \\
Liver & & 0.001 \\
Major histocompatbility complex. class II, DO $\beta$ & -0.45 & 0.002 \\
Lymphotoxin $\beta$ & -0.39 & 0.006 \\
Visceral fat & & 0.36 \\
Contactin 6 & -0.28 & \\
A kinase anchor protein 5 & & \\
\hline
\end{tabular}

A

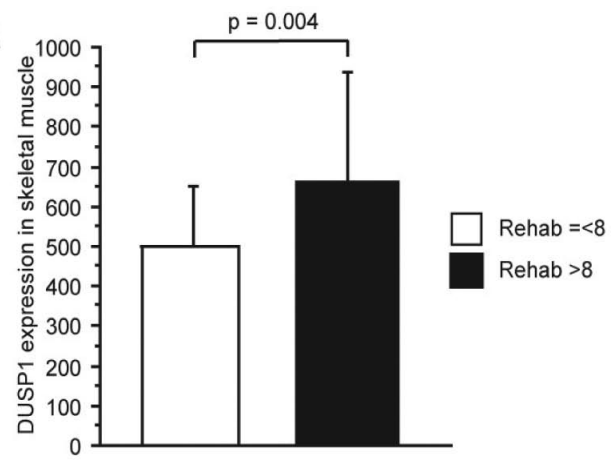

C

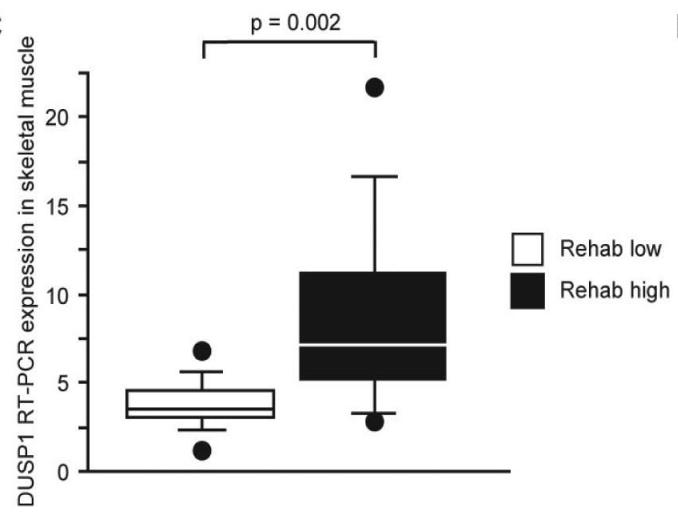

B

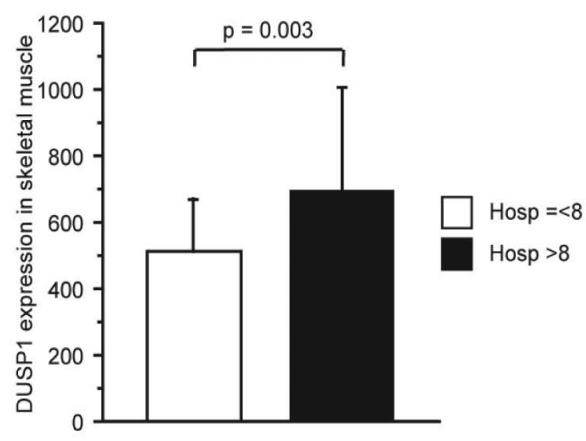

D

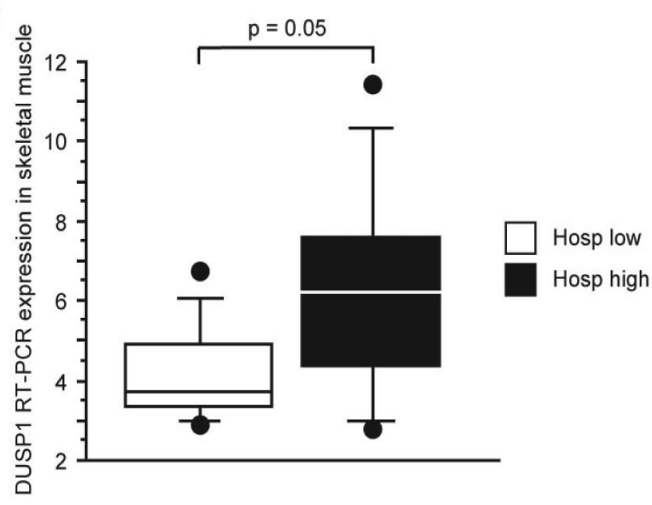

Figure 2. DUSP1 mRNA levels and post-operative stay in the STAGE cohort. DUSP1 mRNA levels in skeletal muscle of all patients with normal ( $\leq 8$ days) and prolonged (>8 days) rehabilitation (A) and hospitalization (B). Box plots of mRNA levels of DUSP1 in skeletal muscle as indicated by RT-PCR in patients with low $(n=13)$ and high $(n=12)$ rehabilitation days $(C)$ and low $(n=10)$ and high $(n=8)$ hospitalization days (D). 'Rehabilitation' includes both days in the hospital and at the rehabilitation homes; 'hospitalization' includes only the days at the hospital. Boxes enclose values between the 25 th and 75 th percentiles. Bars indicate values between the 10th and 90th percentiles. Dots show extreme values. Bar graph plots show the means and SDs.

by RT-PCR (Fig. 2). In a stepwise regression analysis, the correlation with DUSPI was shown to be independent of fasting blood glucose levels and patient age. Interestingly, DUSP1 has previously been highlighted as an early marker of the inflammatory processes and has also been associated with the extent of carotid atherosclerosis (16-19).
We next examined the DUSPI mRNA levels in patients with normal ( $\leq 8$ days) and prolonged ( $>8$ days) rehabilitation and hospitalization. As shown in Fig. 2 DUSP1 mRNA levels were clearly elevated in patients with prolonged rehabilitation ( $p<0.003$, Fig. 2A) as well as hospitalization $(\mathrm{p}<0.004$, Fig. 2B). RT-PCR confirmed the difference in DUSP1 mRNA 

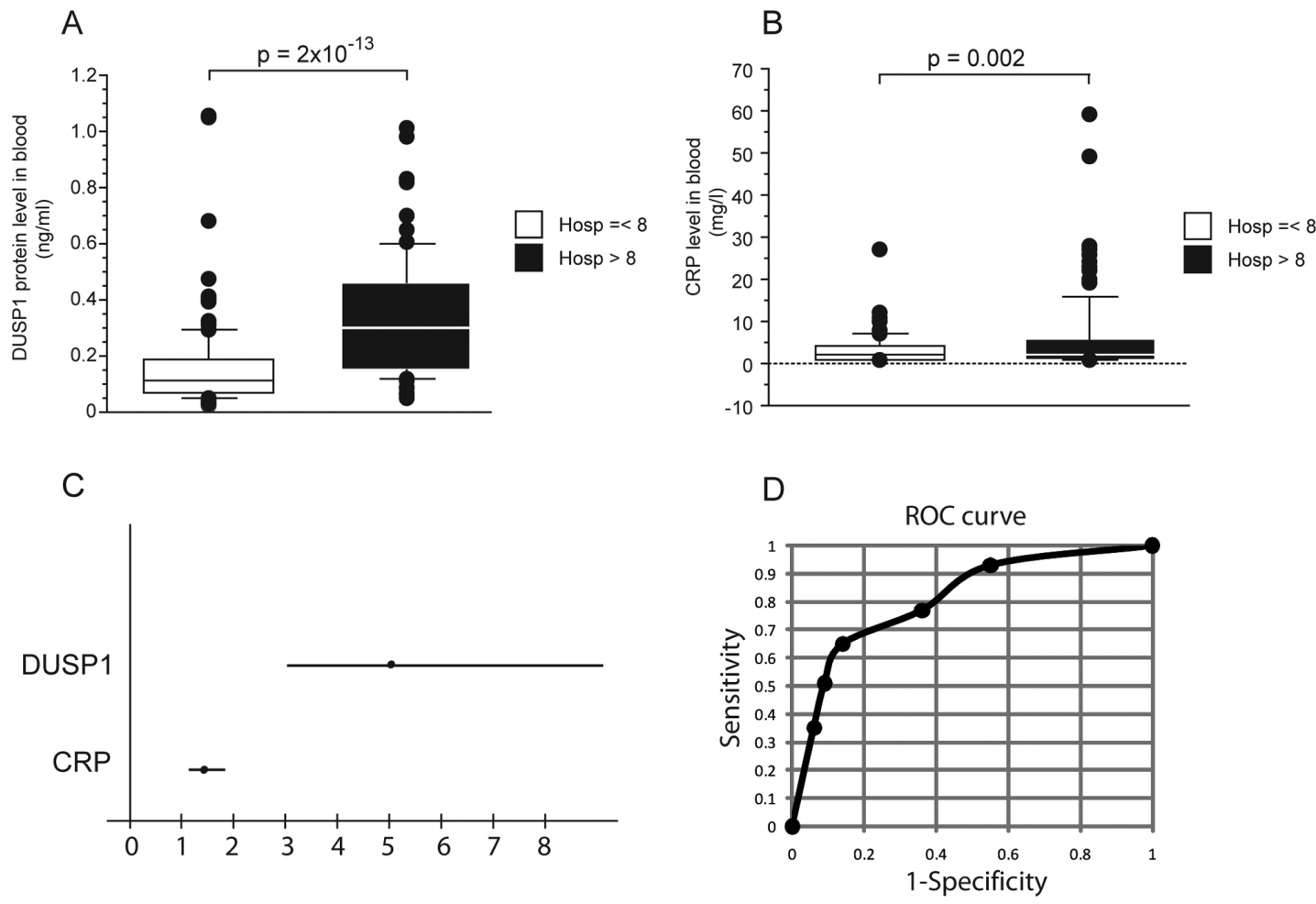

Figure 3. DUSP1 and C-reactive protein (CRP) pre-operative blood levels and hospitalization in a validation CABG cohort. Box plots of DUSP1 (A) and CRP (B) levels in blood drawn pre-operatively in 109 patients hospitalized for $\leq 8$ days and 72 patients hospitalized for $>8$ days. Boxes enclose values between the 25 th and 75th percentiles. Bars indicate values between the 10th and 90th percentiles. Dots show extreme values. Odds ratio (OR) of high $(>8$ days) or low $(\leq 8$ days) hospitalization predicted by DUSP1 [OR=5.1 (CI, 3.0-8.5; $\mathrm{p}<0.0001)]$ and CRP [OR=1.4 (CI, 1.1-1.8; $\mathrm{p}=0.003)]$ (C) ROC curve plotted using sensitivity and specificity calculated from DUSP1 protein levels predicting hospitalization stay after CABG (D).

levels between patients with normal and prolonged rehabilitation and hospitalization (Fig. 2C and D).

Validation of DUSP1 as a biomarker of post-operative complications and hospitalization. Of $\sim 17,000$ genes examined in three separate tissues in the STAGE cohort, DUSPI mRNA levels were the strongest predictor of post-operative complications and subsequent hospitalization. Although this observation was confirmed by RT-PCR, the relation between DUSPI and the post-operative stay may still be a chance observation, given the multiple testing performed $(3 \times 17,000)$. Furthermore, the gene expression in skeletal muscle is not a feasible biomarker although the tissue is easily obtained. Therefore, in order to validate the correlations between the post-operative hospitalization and DUSPI levels in the STAGE cohort, we prospectively collected pre-operative blood samples from patients undergoing elective CABG at the Tartu University Hospital and measured DUSP1 by ELISA.

The distribution of hospital days in the Tartu cohort was similar to that in the STAGE cohort (data not shown). DUSP1 blood levels clearly separated patients with $\leq 8$ hospital days from those with $>8$ days (Fig. $3 \mathrm{~A}$ ). The levels of C-reactive protein (CRP) in pre-operative blood also differed between these groups but with much lower statistical significance (Fig. 3B). These results were also reflected in the odd ratios: 5.1 for DUSP1 [confidence interval (CI), 3.0-8.5; p<0.0001] and 1.4 for CRP (CI, 1.1-1.8; p=0.003) (Fig. 3C). To test the discriminative power of DUSP1 plasma levels to sort patients into groups of prolonged and normal hospitalization times, a receiver operating characteristic (ROC) curve was established based on DUSP1 sensitivity and specificity (Fig. 3D). At a DUSP1 threshold of $0.25 \mathrm{ng} / \mathrm{ml}$, the ROC curve was clearly displaced toward the upper left corner, and the area under the curve was 0.80 . The ROC curve data indicate that this threshold of DUSP1 plasma concentration can be used to identify patients at increased risk of morbidities leading to prolonged hospitalization after CABG.

\section{Discussion}

In this study, analysis of gene expression profiles of liver, skeletal muscle, and visceral fat isolated from 66 STAGE patients during CABG identified the anti-inflammatory gene, DUSP1 as an independent marker of post-operative morbidities leading to prolonged hospitalization. The predictive value of DUSP1 was validated in a prospective study of 181 CABG patients. In that analysis, patients with post-operative complications leading to a prolonged hospital stay had higher pre-operative blood levels of DUSP1 than those who had no complications events and a normal post-operative stay $\left(\mathrm{p}<2 \times 10^{-13}\right)$. The ROC curve indicated that the plasma levels of DUSP1 can serve as a diagnostic test for the risk of postCABG morbidities leading to prolonged hospitalization.

A rising trend in recent decades has been to shorten in-hospital care in favor of health economy savings (20). This trend has perhaps had an adverse effect on these patients who actually need more careful pre-operative preparations and post-operative care. The savings from curtailing in-hospital care may easily be offset by the increased cost for postoperative complications in a few patients. Ideally, patients 
at increased risk of post-operative complications should be identified before surgery. This would be of great benefit for the individual patient and to health economy. Diabetics who undergo surgery are at increased risk of post-operative complications $(3,4,7,8,12,13)$, most likely because of increased inflammation both systemically (21) and in the arterial wall (22). Other markers that can detect early signs of inflammation would be of great value.

Currently, besides biomarkers of general health status (i.e., age, gender, smoking status, and cholesterol and glucose levels), the only established predictor of inflammation in clinical use is CRP. Other inflammatory markers that are not routinely used in the clinic are interleukins (23), lipoprotein-associated phospholipase A2 and myeloperoxidase (24). Our findings suggest that the anti-inflammatory gene DUSP1, previously identified in a screen for blood markers of atherosclerosis $(16,25)$ can be used as a complement to these markers, or possibly by itself, to help identify patients at increased risk of post-operative complications after vascular surgery.

Located on chromosome $5 \mathrm{q} 34$, DUSP1 is a highly conserved gene that encodes a $39 \mathrm{kDa}$ protein expressed in the nucleus. As its name implies, DUSP1 has a dual specificity for tyrosine and threonine and inactivates mitogen-activated protein kinases (MAPKs) (26). MAPKs are part of a phosphorylation signaling pathway that induces a cascade of genes that trigger innate immune responses (17). Regulation of these genes at the transcriptional and post-transcriptional level by DUSP1 affects cell proliferation and differentiation as well as apoptosis. The MAPK pathway has a negative feedback loop that up-regulates DUSP1 in response to pro-inflammatory stimuli, such as oxidative stress and cytokine secretion $(27,28)$.

DUSP1 has been studied in relation to several cancers and independently predicts outcome of carcinomas $(29,30)$. DUSP1 expression is also induced in patients after cardiopulmonary bypass $(19,31)$. The tight regulation of DUSP1 expression is possibly necessary because minor differences appear to have substantial impact on the magnitude of inflammatory reactions. Accordingly, the level of DUSP1 expression varies with the severity of the disease state, such as in tumor cells, carcinomas, and chronic inflammatory diseases in humans $(19,27-31)$. This variation in DUSP1 expression probably reflects its tight transcriptional regulation, which further supports the notion that DUSP1 is an important regulator of the innate immune response to inflammation.

In summary, we identified DUSP1, a blood marker of subtle inflammation, as a potential biomarker of morbidities after invasive surgery resulting in prolonged hospitalization. In support of this notion, atrial fibrillation and infections, the major causes of post-operative complications in CABG patients, are both related to inflammation $(15,32)$. Our findings provide a strong rationale for measuring blood levels of DUSP1 to predict post-operative complications and a prolonged hospital stay and merit further evaluation in larger longitudinal studies.

\section{Acknowledgements}

We thank Stephen Ordway in San Francisco for editorial assistance. This study was supported by grants from the Swedish Research Council (to J.B. and J.S.), the Karolinska Institutet (to
J.B. and J.S.), the Stockholm County Council, an ALF-grant (to J.B.), the Swedish Foundation for Strategic Research (to J.B.and J.T.), the Swedish Heart-Lung Foundation (to J.B.), the King Gustaf V and Queen Victoria Foundation (to J.B.), the Swedish Society of Medicine (to J.B., J.S. and J.T.), the Hans and Loo Osterman Foundation for Geriatric Research (to J.S.and J.B.), the Wennergren Foundation (to J.T.), The Tornspiran and the Mats Klebergs Foundations (to T.A.and T.I.), the Estonian Science Foundation (to A.R.), Vinnova Sweden-Japan (to J.B. and J.T.), a Vinnova research grant (to J.T. and J.B.), the PhD Programme in Medical Bioinformatics (to S.H., J.B. and J.T.), the Carl Tryggers Foundation (to J.T.) and the Clinical Gene Networks (to J.B. and J.T.).

\section{References}

1. Schadt EE, Sachs A and Friend S: Embracing complexity, inching closer to reality. Sci STKE 2005: pe40, 2005.

2. Ouzounian M, Lee DS, Gramolini AO, Emili A, Fukuoka M and Liu PP: Predict, prevent and personalize: Genomic and proteomic approaches to cardiovascular medicine. Can J Cardiol 23 (Suppl A): 28A-33A, 2007.

3. Alserius T, Hammar N, Nordqvist $\mathrm{T}$ and Ivert $\mathrm{T}$ : Risk of death or acute myocardial infarction 10 years after coronary artery bypass surgery in relation to type of diabetes. Am Heart J 152: 599-605, 2006.

4. Latham R, Lancaster AD, Covington JF, Pirolo JS and Thomas CS: The association of diabetes and glucose control with surgical-site infections among cardiothoracic surgery patients. Infect Control Hosp Epidemiol 22: 607-612, 2001.

5. Pomposelli JJ, Baxter JK, III, Babineau TJ, et al: Early postoperative glucose control predicts nosocomial infection rate in diabetic patients. JPEN J Parenter Enteral Nutr 22: 77-81, 1998.

6. Takamura T, Sakurai M, Ota T, Ando H, Honda M and Kaneko S: Genes for systemic vascular complications are differentially expressed in the livers of type 2 diabetic patients. Diabetologia 47: 638-647, 2004.

7. Umpierrez GE, Isaacs SD, Bazargan N, You X, Thaler LM and Kitabchi AE: Hyperglycemia: an independent marker of in-hospital mortality in patients with undiagnosed diabetes. J Clin Endocrinol Metab 87: 978-982, 2002.

8. Anderson RE, Klerdal K, Ivert T, Hammar N, Barr G and Owall A: Are even impaired fasting blood glucose levels preoperatively associated with increased mortality after CABG surgery? Eur Heart J 26:1513-1518, 2005.

9. Hagg S, Skogsberg J, Lundstrom J, et al: Multi-organ expression profiling uncovers a gene module in coronary artery disease involving transendothelial migration of leukocytes and LIM domain binding 2: the Stockholm Atherosclerosis Gene Expression (STAGE) study. PLoS Genet 5: e1000754, 2009.

10. Carlson K: Lipoprotein fractionation. J Clin Pathol Suppl (Assoc Clin Pathol) 5: 32-37, 1973.

11. Irizarry RA, Bolstad BM, Collin F, Cope LM, Hobbs B and Speed TP: Summaries of Affymetrix GeneChip probe level data. Nucleic Acids Res 31: e15, 2003.

12. Corsetti JP, Zareba W, Moss AJ and Sparks CE: Serum glucose and triglyceride determine high-risk subgroups in non-diabetic postinfarction patients. Atherosclerosis 183: 293-300, 2005.

13. Szabo Z, Hakanson E and Svedjeholm R: Early postoperative outcome and medium-term survival in 540 diabetic and 2239 nondiabetic patients undergoing coronary artery bypass grafting. Ann Thorac Surg 74: 712-719, 2002.

14. Issac TT, Dokainish H and Lakkis NM: Role of inflammation in initiation and perpetuation of atrial fibrillation: a systematic review of the published data. J Am Coll Cardiol 50: 2021-2028, 2007.

15. Liuba I, Ahlmroth H, Jonasson L, et al: Source of inflammatory markers in patients with atrial fibrillation. Europace 10: 848-853, 2008.

16. Kang JG, Patino WD, Matoba S and Hwang PM: Genomic analysis of circulating cells: a window into atherosclerosis. Trends Cardiovasc Med 16: 163-168, 2006.

17. Lang R, Hammer M and Mages J: DUSP meet immunology: dual specificity MAPK phosphatases in control of the inflammatory response. J Immunol 177: 7497-7504, 2006. 
18. Ruel M, Bianchi C, Khan TA, et al: Gene expression profile after cardiopulmonary bypass and cardioplegic arrest. J Thorac Cardiovasc Surg 126: 1521-1530, 2003

19. Khan TA, Bianchi C, Ruel M, et al: Mitogen-activated protein kinase inhibition and cardioplegia-cardiopulmonary bypass reduce coronary myogenic tone. Circulation 108 (Suppl 1): II348-II353, 2003.

20. Cowper PA, DeLong ER, Hannan EL, et al: Is early too early? Effect of shorter stays after bypass surgery. Ann Thorac Surg 83: 100-107, 2007.

21. Schalkwijk CG, Poland DC, van Dijk W, et al: Plasma concentration of C-reactive protein is increased in type I diabetic patients without clinical macroangiopathy and correlates with markers of endothelial dysfunction: evidence for chronic inflammation. Diabetologia 42: 351-357, 1999.

22. Kanter JE, Averill MM, Leboeuf RC and Bornfeldt KE: Diabetes-accelerated atherosclerosis and inflammation. Circ Res 103: e116-e117, 2008.

23. Fisman EZ, Adler Y and Tenenbaum A: Biomarkers in cardiovascular diabetology: interleukins and matrixins. Adv Cardiol 45: 44-64, 2008

24. Virani SS, Polsani VR and Nambi V: Novel markers of inflammation in atherosclerosis. Curr Atheroscler Rep 10: 164-170, 2008.

25. Patino WD, Mian OY, Kang JG, et al: Circulating transcriptome reveals markers of atherosclerosis. Proc Natl Acad Sci USA 102: 3423-3428, 2005.
26. Alessi DR, Smythe C and Keyse SM: The human CL100 gene encodes a Tyr/Thr-protein phosphatase which potently and specifically inactivates MAP kinase and suppresses its activation by oncogenic ras in Xenopus oocyte extracts. Oncogene 8: 2015-2020, 1993.

27. Abraham SM and Clark AR: Dual-specificity phosphatase 1: a critical regulator of innate immune responses. Biochem Soc Trans 34: 1018-1023, 2006.

28. Chi H, Barry SP, Roth RJ, et al: Dynamic regulation of pro- and anti-inflammatory cytokines by MAPK phosphatase 1 (MKP-1) in innate immune responses. Proc Natl Acad Sci USA 103: 2274-2279, 2006.

29. Denkert C, Schmitt WD, Berger S, et al: Expression of mitogenactivated protein kinase phosphatase-1 (MKP-1) in primary human ovarian carcinoma. Int J Cancer 102: 507-513, 2002.

30. Tsujita E, Taketomi A, Gion T, et al: Suppressed MKP-1 is an independent predictor of outcome in patients with hepatocellular carcinoma. Oncology 69: 342-347, 2005.

31. Khan TA, Bianchi C, Araujo EG et al: Cardiopulmonary bypass reduces peripheral microvascular contractile function by inhibition of mitogen-activated protein kinase activity. Surgery 134: 247-254, 2003

32. Bruins P, te Velthuis H, Yazdanbakhsh AP, et al: Activation of the complement system during and after cardiopulmonary by pass surgery: postsurgery activation involves $\mathrm{C}$-reactive protein and is associated with postoperative arrhythmia. Circulation 96: 3542-3548, 1997. 